\title{
Repaglinida, un nuevo antidiabético oral
}

\author{
J. Ma. Calvo Romero, E. Mà . L ima RodríGuez \\ Servicio de Medicina Interna. \\ Unidad Docente de Medicina Familiar y Comunitaria. \\ Hospital Regional Universitario Infanta Cristina. Badajoz.
}

\section{Repaglinide, a new oral antidiabetic agent}

\section{RESUMEN}

En los últimos años el arsenal de fármacos orales útiles en el tratamiento de la diabetes mellitus tipo 2 se ha ampliado. La repaglinida es el primero de una nueva clase de antidiabéticos orales derivados del ácido benzoico, no pertenecientes al grupo de las sulfonilureas, con acción estimuladora de la secreción de insulina. La repaglinida mejora la regulación de la glucemia prandial, al corregir la secreción disminuida y retrasada de insulina relacionada con las comidas en los diabéticos tipo 2. Utilizada en monoterapia tiene una eficacia similar a las sulfonilureas o a la metformina en reducir la glucemia basal y la hemoglobina glucosilada.

Palabras clave: Repaglinida. Antidiabéticos orales. Diabetes mellitus tipo 2.

\section{INTRODUCCIÓN}

Los pacientes con diabetes mellitus (DM) tipo 2 presentan diferentes grados de resistencia a la insulina y una función alterada de la célula beta pancreática con el consiguiente déficit de insulina ${ }^{1}$. No se conoce qué evento, la resistencia a la insulina o el déficit de insulina, inicia la cascada que finalmente lleva a una DM tipo 2 manifiesta ${ }^{1-3}$. La repaglinida es el primero de una nueva clase de antidiabéticos orales derivados del ácido benzoico, no pertenecientes al grupo de las sulfonilureas, que estimula la secreción de insulina, y que ha demostrado su eficacia en el tratamiento de la DM tipo $2^{4,5}$. En este trabajo revisamos las características y eficacia de la repaglinida.

\begin{abstract}
The arsenal of oral agents with usefulness in the treatment of type 2 diabetes mellitus has been extended in the last few years. Repaglinide is the first of a new class of oral antidiabetic agents that are benzoic acid derivatives, which is a nonsulfonylurea insulin secretagogue. Repaglinide improves the prandial glucose regulation, restoring the diminished and delayed insulin secretion related with the meals in type 2 diabetic patients. When used as monotherapy, the decreases in fasting plasma glucose and glycosylated hemoglobin is similar to those observed with sulfonylureas or metformin.
\end{abstract}

Key words: Repaglinide. Oral antidiabetic agents. Type 2 diabetes mellitus. 
rivado del ácido benzoico estructuralmente relacionado con la meglitinida, no perteneciente al grupo de las sulfonilureas, que estimula la secreción de insulina, mejorando la $\mathrm{RGP}^{4,9,10}$. Tiene una configuración en forma de $\mathrm{U}$ similar a la que presentan sulfonilureas como la glibenclamida y la glimepiri$\mathrm{da}^{11}$. Se une al menos a 2 receptores de la célula beta pancreática ${ }^{12}$. Uno de ellos es un receptor con una alta afinidad por la repaglinida y una baja afinidad por las sulfonilureas, y el otro es un receptor con una alta afinidad por las sulfonilureas y una baja afinidad por la repaglinida ${ }^{12}$. La repaglinida causa el cierre de los canales de potasio ATP-sensibles de la célula beta pancreática ${ }^{13,14}$. Esto da lugar a la despolarización de la célula beta y a la apertura de los canales de calcio voltaje-sensibles, que permite el flujo hacia el interior celular de iones calcio extracelulares. El aumento del calcio intracelular estimula la secreción de insulina. No obstante, se ha sugerido que la capacidad ionoforética de la repaglinida puede no ser un mecanismo determinante en su acción liberadora de insulina ${ }^{15}$. La repaglinida tiene una acción insulinotrópica ${ }^{16}$, aumenta la secreción de insulina y de somatostatina, pero no causa una indeseable estimulación de la liberación de glucagón ${ }^{17}$. No estimula la liberación de insulina en ausencia de glucosa exógena y no inhibe la biosíntesis de proinsulina ${ }^{5,12-14}$. Se desconoce si el efecto estimulador de la secreción de insulina de la repaglinida es aditivo al de las sulfonilureas.

\section{FARMACOCINÉTICA}

La repaglinida administrada por vía oral es rápidamente absorbida en 30-60 minutos, y presenta una rápida eliminación plasmática, con una vida media plasmática de aproximadamente 1 hora ${ }^{18,19}$. Es inactivada en el hígado, y más del $90 \%$ se excreta en la bilis en forma de metabolitos inacti$\operatorname{vos}^{19,20}$. El principal metabolito es el ácido dicar boxílico ${ }^{21}$. Una dosis de $2 \mathrm{mg}$ de repaglinida 4 veces al día no da lugar a acumulación ${ }^{21}$. Por su conducta farmacocinética, la repaglinida causa una rápida pero breve liberación de insulina. La farmacocinética en los ancianos es similar ${ }^{19,20}$.

\section{EFICACIA CLÍNICA}

La repaglinida utilizada en monoterapia tiene una eficacia similar a las sulfonilureas o a la metformina en reducir la glucemia basal y la hemoglobina glucosilada (HbA1c). En diabéticos tipo 2 tratados previamente únicamente con dieta, la repaglinida reduce la glucemia basal en 50-60 $\mathrm{mg} / \mathrm{dl}$ y la HbA1c en 1,7-1,9 puntos $^{22,23}$. En un estudio multicéntrico randomizado a doble ciego con un año de seguimiento de 576 pacientes con DM ti- po 2 de más de 6 meses de evolución desde el diagnóstico, la repaglinida mostró una eficacia similar a la gliburida, disminuyendo la glucemia basal 34-47 mg/dl y la HbA1c 1,5-1,8 puntos $^{24}$. En otro estudio multicéntrico randomizado a doble ciego con un año de seguimiento de 424 diabéticos tipo 2 con una media de 8 años desde el diagnóstico de su DM y en su mayor parte tratados previamente con antidiabéticos orales, la HbA1c sólo disminuyó 0,58 puntos en los tratados con repaglinida, descenso similar al conseguido con la gliburida, con una incidencia similar de hipoglucemia en ambos grupos ${ }^{25}$. En este mismo estudio, en los pacientes previamente tratados únicamente con dieta, la HbA1c disminuyó 2,4 puntos en el grupo de la gliburida y 1 punto en el grupo de la repaglinida $^{25}$.

En un estudio de 12 semanas comparando la repaglinida y la glibenclamida en 44 diabéticos tipo 2 tratados previamente con sulfonilureas, la repaglinida disminuyó más la glucemia postprandial pero menos la glucemia basal que la glibenclamida ${ }^{26}$. En otro estudio de 14 semanas de 195 pacientes con DM tipo 2 previamente tratados con antidiabéticos orales, la repaglinida fue similar en tolerancia y eficacia a la glibenclamida, con la salvedad de que la repaglinida redujo más la glucemia 2 horas postprandial (147 mg/dl vs $165 \mathrm{mg} / \mathrm{dl}$ ), aunque sin alcanzar significación estadística ${ }^{27}$. Otro estudio randomizado de 18 semanas demostró la eficacia de la repaglinida frente a placebo en reducir la glucemia basal y postprandial ${ }^{28}$. La repaglinida no modifica de manera significativa los niveles plasmáticos de lípidos ${ }^{22-28}$.

El efecto de la repaglinida es aditivo al de la metformina. En diabéticos tipo 2 tratados previamente con metformina con un control metabólico inadecuado, la asociación de repaglinida consiguió reducir la glucemia basal $40 \mathrm{mg} / \mathrm{dl}$ y la $\mathrm{HbA} 1 \mathrm{c} 1,4$ puntos, siendo esta asociación superior en eficacia a la monoterapia con metformina o repaglinida ${ }^{29}$.

\section{INDICACIONES}

La repaglinida ha sido recientemente comercializada en España. Sus indicaciones son similares a las de las sulfonilureas. La repaglinida está indicada en el tratamiento de pacientes con DM tipo 2 en los que la dieta y el ejercicio físico no consiguen un control adecuado de la glucemia ${ }^{4,30}$. Es unas 2 veces más cara que las sulfonilureas de segunda generación y algo más cara que la metformina.

La asociación de repaglinida y metformina puede conseguir un control metabólico adecuado en pacientes con DM tipo 2 no controlados con monoterapia $^{29}$. No existen datos por el momento sobre la eficacia y seguridad de la asociación de la repagli- 
Tabla I

COMPARACIÓN DE LAS CARACTERÍSTICAS DE LOS FÁRMACOS ORALES UTILIZADOS EN EL TRATAMIENTO DE LA DIABETES MELLITUS TIPO 2

\begin{tabular}{|c|c|c|c|c|}
\hline & Sulfonilureas & Metformina & Acarbosa & Repaglinida \\
\hline $\begin{array}{l}\text { Mecanismos de } \\
\text { acción }\end{array}$ & $\begin{array}{l}\text { Aumentan la } \\
\text { secreción de } \\
\text { insulina }\end{array}$ & $\begin{array}{l}\text { Disminuye la } \\
\text { producción hepática } \\
\text { de glucosa, aumenta } \\
\text { la sensibilidad a la insulina }\end{array}$ & $\begin{array}{l}\text { Enlentece la } \\
\text { absorción } \\
\text { gastrointestinal } \\
\text { de glucosa }\end{array}$ & $\begin{array}{l}\text { Aumenta la } \\
\text { secreción de } \\
\text { insulina }\end{array}$ \\
\hline $\begin{array}{l}\text { Disminución de la } \\
\text { glucemia basal } \\
\text { (mg/dl) }\end{array}$ & $60-70$ & $60-70$ & $20-30$ & $50-60$ \\
\hline $\begin{array}{l}\text { Disminución de la } \\
\text { HbAlc (puntos de \%) }\end{array}$ & $1,5-2$ & $1,5-2$ & $0,7-1$ & $1,7-1,9$ \\
\hline Triglicéricos & Sin efecto & Disminución & Disminución leve & Sin efecto \\
\hline Colesterol-HDL & Sin efecto & Aumento ligero & Sin efecto & Sin efecto \\
\hline Colesterol-LDL & Sin efecto & Disminución & Sin efecto & Sin efecto \\
\hline Peso corporal & Aumento & Disminución & Sin efecto & Aumento \\
\hline Insulina plasmática & Aumento & Disminución & Sin efecto & Aumento \\
\hline Efectos adversos & Hipoglucemia & $\begin{array}{l}\text { Digestivos, } \\
\text { acidosis láctica (rara) }\end{array}$ & Digestivos & Hipoglucemia \\
\hline Coste & Menor coste & $\begin{array}{l}2 \text { veces el de las } \\
\text { sulfonilureas }\end{array}$ & $\begin{array}{l}2 \text { veces el de las } \\
\text { sulfonilureas }\end{array}$ & $\begin{array}{l}2 \text { veces el de las } \\
\text { sulfonilureas }\end{array}$ \\
\hline
\end{tabular}

nida con sulfonilureas, acarbosa o insulinoterapia.

La repaglinida puede utilizarse en alérgicos a sulfamidas, a diferencia de las sulfonilureas. Debido a su farmacocinética, la repaglinida no está contraindicada en pacientes con insuficiencia renal, al contrario que las sulfonilureas. En pacientes con hepatopatía, se recomienda una titulación de la dosis más lenta.

\section{DOSIFICACIÓN Y EFECTOS SECUNDARIOS}

La dosis de inicio de la repaglinida es $0,5 \mathrm{mg}$ vía oral, tomada 15 minutos antes de cada comida. En pacientes previamente tratados con otros antidiabéticos orales sin un control adecuado, se puede comenzar con una dosis de 1-2 mg antes de cada comida. La dosis máxima es $4 \mathrm{mg}$ antes de cada comida en hasta un máximo de 4 comidas al día (16 mg/día). El aumento de la dosis se puede realizar semanalmente. Una dosis de $1 \mathrm{mg}$ de repaglinida tres veces al día produce un $90 \%$ del efecto máximo reductor de la glucemia ${ }^{22}$. El tratamiento con una dosis de repaglinida antes de cada comida consigue un control glucémico similar independientemente del número de comidas realizadas al día, es bien tolerado, y permite un estilo de vida más flexible a los pacientes diabéticos tipo $2^{31}$.

La hipoglucemia y el aumento de peso son los únicos efectos adversos que se presentan con mayor frecuencia en los pacientes tratados con repaglinida que en los que reciben placebo ${ }^{32}$. Probable- mente, la repaglinida causa menos hipoglucemias y de menor severidad que las sulfonilureas ${ }^{22-27}$. El riesgo de hipoglucemia en los diabéticos tipo 2 tratados con repaglinida que omiten o retrasan una comida parece ser menor que en los tratados con sulfonilureas. En un estudio de 43 pacientes, los diabéticos tratados con gliburida que suprimieron una comida presentaron un descenso de la glucemia mínima de 77 a $61 \mathrm{mg} / \mathrm{dl}$, mientras que en los tratados con repaglinida no hubo cambios en la glucemia mínima ${ }^{33}$. La repaglinida se asocia a un aumento de peso de aproximadamente un $3 \%^{22-28}$. En pacientes tratados previamente con sulfonilureas, el cambio a repaglinida no causa aumento de peso $^{24}$.

\section{CONCLUSIONES}

La repaglinida es un fármaco eficaz y seguro en el tratamiento de la DM tipo 2. La eficacia de la repaglinida en reducir la glucemia basal y la hemoglobina glucosilada es similar a la de las sulfonilureas o la metformina. Puede utilizarse en alérgicos a sulfamidas, a diferencia de las sulfonilureas. Posi bles ventajas de la repaglinida frente a las sulfonilu reas son un menor riesgo de hipoglucemia en los pacientes que omiten o retrasan una comida, y un menor riesgo de hipoglucemia en general, por lo que podría estar especialmente indicada en pacientes diabéticos tipo 2 poco disciplinados o con dificultades para seguir un régimen regular de comidas. 
En la tabla I se comparan las características de la repaglinida con los otros antidiabéticos orales disponibles en España. La repaglinida es una buena opción terapéutica a considerar en la gran población de diabéticos tipo 2 que atendemos tanto en Atención Especializada como en Atención Primaria.
CORRESPONDENCIA:

J. $\mathrm{M}^{\mathrm{a}}$. Calvo Romero

Héroes de Cascorro 9-3ำA

06004 Badajoz

\section{Bibliografía}

1. DeFronzo RA, Bonadonna RC, Ferrannini E. Pathogenesis of non-insulin dependent diabetes mellitus (NIDDM): a balanced overview. Diabetes Care 1992; 15: 318-68.

2. Polonsky KS. Lilly Lecture 1994 . The b-cell in diabetes: from molecular genetics to clinical research. Diabetes 1995; 44: 705-17.

3. Saad MF, Knowler WC, Pettitt DJ, Nelson RG, Mott $\mathrm{DM}$, Bennett $\mathrm{PH}$. Sequential changes in serum insulin concentration during development of non-insulindependent diabetes. Lancet 1989; 1: 1356-9.

4. Malaisse WJ. Stimulation of insulin release by nonsulfonylurea hypoglycemic agents: the meglitinide family. Horm Metab Res 1995; 27: 263-6.

5. DeFronzo RA. Pharmacologic therapy for type 2 diabetes mellitus. An Intern Med 1999; 131: 281-303.

6. King GL, Banskota NK. Mechanisms of diabetic microvascular complications. En: Khan CR, Weir GC, eds. Joslin's Diabetes Mellitus (13aㅡ ed.). Philadelphia: Lea \& Febiger 1994; 631-47.

7. Chait A, Bierman EL. Pathogenesis of macrovascular disease in diabetes. En: Khan CR, Weir GC, eds. Joslin's Diabetes Mellitus (13 ${ }^{\underline{a}}$ ed.). Philadelphia: Lea \& Febiger 1994; 648-59.

8. Lefebvre PJ, Scheen AJ. Glucose metabolism and the postprandial state. Eur J Clin Invest 1999; 29 (Suppl 2): 1-6.

9. Owens DR. Repaglinide-prandial glucose regulator: a new class of oral antidiabetic drugs. Diabet Med 1998; 15 (Suppl 4): 28-36.

10. Mooradian AD, Thurman JE. Drug therapy of postprandial hyperglycaemia. Drugs 1999; 57: 19-29.

11. Lins L, Brasseur R, Malaisse WJ. Conformational analysis of non-sulfonylurea hypoglycemic agents of the meglitinide family. Biochem Pharmacol 1995; 50: 1879-84.

12. Fuhlendorff $\mathrm{J}$, Rorsman $\mathrm{P}$, Kofod $\mathrm{H}$, Brand $\mathrm{CL}$, Rolin $B$, Mackay $P$, et al. Stimulation of insulin release by repaglinide and glibenclamide involves both common and distinct processes. Diabetes 1998, 47: 345-51.

13. Malaisse WJ. Repaglinide, a new oral antidabetic agent: a review of recent preclinical studies. Eur J Clin Invest 1999; 29 (Suppl 2): 21-9.

14. Malaisse WJ. Mechanism of action of a new class of insulin secretagogues. Exp Clin Endocrinol Diabetes 1999; 107 (Suppl 4): 140-3.

15. Bakkali-Nadi A, Malaisse-Lagae F, Malaisse WJ. Ionophoretic activity of meglitinide analogues. Diabetes Res 1994; 27: 61-71.

16. Bakkali-Nadi A, Malaisse-Lagae F, Malaisse WJ. Insulinotropic action of meglitinide analogs: concentration-response relationship and nutrient dependency. Diabetes Res 1994; 27: 81-7.

17. Leclercq-Meyer V, Ladriere L, Fuhlendorff J, Malaisse WJ. Stimulation of insulin and somatostatin release by two meglitinides analogs. Endocrine 1997; 7: 311-7.
18. Hatorp V, Oliver S, Su CA. Bioavailability of repaglinide, a novel antidiabetic agent, administered orally in tablet or solution form or intravenously in healthy male volunteers. Int J Clin Pharmacol Ther 1998; 36: 63641.

19. Hatorp V, Huang WC, Strange P. Repaglinide pharmacokinetics in healthy young adult and elderly subjects. Clin Ther 1999; 21: 702-10.

20. Hatorp V, Huang WC, Strange P. Pharmacokinetic profiles of repaglinide in elderly subjects with type 2 diabetes. J Clin endocrinol Metab 1999; 84: 1475-8.

21. van Heiningen PN, Hatorp V, Kramer Nielsen K, Hansen KT, van Lier JJ, De Merbel NC, et al. Absorption, metabolism and excretion of a single oral dose of (14)C-repaglinide during repaglinide multiple dosing. Eur J Clin Pharmacol 1999; 55: 521-5.

22. Schwartz SL, Goldberg RB, Strange P. Repaglinide in type 2 diabetes: a randomized, double blind, placebocontrolled, dose-response study. Repaglinide Study Group (Abstract). Diabetes 1998; 47 (Suppl 1): A98.

23. Berger $S$, Strange $P$. Repaglinide, a novel and hypoglycemic agent in type 2 diabetes mellitus: a randomized, placebo-controlled, double-blind, fixed-dose study. Repaglinide Study Group (Abstract). Diabetes 1998; 47 (Suppl 1): A18

24. Marbury T, Huang WC, Strange P, Lebovitz H. Repaglinide versus glyburide: a one-year comparison trial. Diabetes Res Clin Pract 1999; 43: 155-66.

25. Wolffenbuttel BH, Landgraf R. A 1-year multicenter randomized double-blind comparison of repaglinide and glyburide for the treatment of type 2 diabetes. Dutch and German Repaglinide Study Group. Diabetes Care 1999; 22: 463-7.

26. Wolffenbuttel BH, Nijst L, Sels JP, Menheere PP, Muller PG, Kruseman AC. Effects of a new oral hypoglycaemic agent, repaglinide, on metabolic control in sulphonylurea-treated patients with NIDDM. Eur J Clin Pharmacol 1993; 45: 113-6.

27. Landgraf R, Bilo HJ, Muller PG. A comparison of repaglinide and glibenclamide in the treatment of type 2 diabetic patients previously treated with sulphonylureas. Eur J Clin Pharmacol 1999; 55: 165-71.

28. Goldberg RB, Einhorn D, Lucas CP, Rendell MS, Damsbo P, Huang WC, et al. A randomized placebocontrolled trial of repaglinide in the treatment of tye 2 diabetes. Diabetes Care 1998; 21: 1897-903.

29. Moses R, Slobodniuk R, Boyages S, Colagiuri S, Kidson W, Carter J, et al. Effect of repaglinide addition to metformin monotherapy on glycemic control in patients with type 2 diabetes. Diabetes Care 1999; 22: 119-24.

30. Gomis R. Repaglinide as monotherapy in type 2 diabetes. Exp Clin Endocrinol Diabetes 1999; 107 (Suppl 4): $133-5$

31. Damsbo P, Marbury TC, Hatorp V, Clauson P, Muller $P G$. Flexible prandial glucose regulation with repagli- 